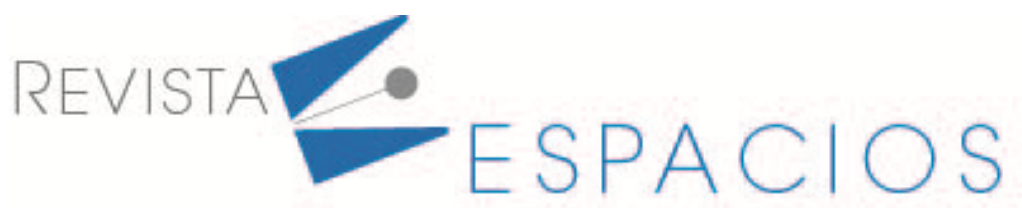

\title{
Responsabilidad social empresarial: una experiencia regional en las empresas del sector servicios
}

\section{Corporate Social Responsibility: a regional experience in companies in the Service Sector}

\author{
RODRIGUEZ, Magda ${ }^{1}$ \\ PEÑARANDA, Marta ${ }^{2}$ \\ MANZANO, Omaira ${ }^{3}$
}

\begin{abstract}
Resumen
La investigación tuvo como propósito determinar las prácticas de Responsabilidad Social en las empresas del sector servicios en la ciudad de Ocaña (Colombia), mediante una investigación con enfoque cuantitativo de tipo transversal descriptivo apoyada en la herramienta ISO 26000. Los resultados evidenciaron que dichas empresas enfocan su compromiso en las dimensiones prácticas justas de operación, asuntos de consumidores, gobernanza y derechos Humanos. El ANOVA comprobó que el nivel de formación del gerente y el tamaño de la empresa inciden en la gestión de la RSE. Palabras clave: Responsabilidad social empresarial, empresa de servicios, ISO 26000, Análisis de varianza

Abstract

The purpose of the research was to determine the practices of social responsibility in companies of the service sector in the city of Ocaña (Colombia), through an investigation with a quantitative approach of a descriptive transversal type supported by the ISO 26000 tool. The results could show that said companies focus their commitment on fair operating practices, consumer affairs, governance and Human Rights. The ANOVA verified that the level of management training and the size of the company affect the management of CSR.

key words: Corporate social responsibility, service companies, ISO 26000, analysis of variance
\end{abstract}

\section{Introducción}

La Responsabilidad Social Empresarial (RSE) como campo de estudio ha sido analizada desde varias posturas. Segun Friedman (1962), una empresa responsable debe estar centrada en la generación de riqueza, siempre y cuando esta se encuentre enmarcada en el cumplimiento de la normatividad legal a la cual está sometida. Dicha postura ha evolucionado sin dejar de lado el beneficio económico para la empresa y sus accionistas, las organizaciones que actúan bajo las prácticas responsables direccionan sus estrategias y procesos

\footnotetext{
${ }^{1}$ Magister en Administración de las Organizaciones. Investigadora del Grupo GIDSE. Docente Universidad Francisco de Paula Santander Ocaña. (UFPSO) Correo electrónico:mmrodriguezc@ufpso.edu.co

${ }^{2}$ Magister en Administración de las Organizaciones. Investigadora del Grupo GIDSE. Docente Universidad Francisco de Paula Santander Ocaña. (UFPSO). Correo electrónico:mmpenarandap@ufpso.edu.co

${ }^{3}$ Doctora en Administración de Negocios (DBA). Investigadora del Grupo GRINDES. Docente Asistente, Universidad Nacional Abierta y a Distancia (UNAD), Ocaña - Colombia. Correo electrónico: omaira.manzano@unad.edu.co
} 
organizacionales no solo respecto a la dimensión económica, sino también la social y medio ambiental, es decir que vaya más allá de las pretensiones de maximizar la riqueza de la empresa (Rojas \& Madero, 2018).

Dentro de la nueva perspectiva de la responsabilidad social se hace necesario comunicar a sus grupos de interés una información integral que contemple lo económico, social y ambiental, es así como Strandberg (2010), señala que las organizaciones no solo deben rendir informes sobre sus asuntos económicos, sino que, de igual forma, contemplen aspectos relacionados con la ejecución de su objeto empresarial, enmarcadas en las prácticas responsables, producto de las demandas de los stakeholder o grupos de interés.

Desde la proposición anterior se considera que las empresas deben incursionar en el empleo de mejores prácticas encaminadas al cumplimiento de su Responsabilidad Social, de modo que satisfagan las necesidades de sus grupos de interés, garantizando una imagen más aceptable y comprometida con la sustentabilidad. Se agrega valor a las organizaciones y se genera una ventaja competitiva para lograr el posicinamiento en los exigentes mercados internacionales, que ven la Responsabilidad Social como una política organizacional de contribución al bienestar de la sociedad y sostenibilidad empresarial.

El presente estudio aborda la caracterización de las prácticas de Responsabilidad Social Empresarial en las pequeñas y medianas empresas del sector Servicios. Teniendo en cuenta que este sector económico es de gran importancia desde su contribución al desarrollo local, regional y nacional. Aquí, la Responsabilidad Social se convierte en un desafío, ya que las empresas del sector se desenvuelven en un contexto complejo caracterizado por mercados globalizados, problemáticas sociales, reformas tributarias, apertura de mercados, limitaciones para acceder a mercados internacionales, entre otras. Para sobresalir en estas dinámicas y lograr la competitividad deben superar estos obstáculos y no solo pensar en conseguir beneficios económicos, sino articular su labor productiva con políticas que generen beneficios a la comunidad donde se desenvuelven.

El sector servicios se caracteriza por su dinamismo y expansión en la estructura productiva de las economías a nivel mundial (Valotto, 2007). Así mismo, la Organización Mundial de Comercio, OMC, mediante el Acuerdo General sobre el Comercio de Servicios (AGCS), expresa el rápido crecimiento del sector en la economía mundial. De ahí la importancia de abordar el estudio del sector desde una mirada regional, ya que en la ciudad de Ocaña, según el censo Empresarial realizado por la Universidad Francisco de Paula Santander Ocaña y la Cámara de Comercio (2010), el $22.8 \%$ de los establecimientos pertenecen al sector servicios. Además, es necesario abordar este tipo de investigaciones en pequeñas empresas pues se evidencia un vacío en el conocimiento debido a que la mayoría de estudios son realizados en grandes organizaciones.

\section{Metodología}

Como método de investigación, se adopta un diseño con enfoque cuantitativo no experimental de tipo trasversal descriptivo. Se recolectan datos en un solo momento del tiempo buscando las particularidades, propiedades y tipologías importantes del objeto de estudio (Hernández, Fernández y Baptista, 2010). Se utilizó como instrumento un cuestionario tipo escala de Likert, cuyas preguntas corresponden a las dimensiones adaptadas de la herramienta ISO 26000 diseñada para la evaluación de la Responsabilidad Social Empresarial donde se evalúan siete dimensiones de responsabilidad social. Este instrumento fue aplicado a 117 Directivos de las pequeñas y medianas empresas objeto de estudio.

La muestra estuvo conformada principalmente por empresas de transporte, inmobiliarias, contratación de personal temporal, servicios generales (mantenimiento, aseo, casinos y restaurantes), agremiaciones económicas, publicidad, informática y gestión de proyectos, servicios de tramitología, recaudo de servicios públicos y salas de belleza. 
EL instrumento fue validado por tres expertos y se midió la confiabilidad por medio del coeficiente Alfa Crombach del $83.5 \%$. Por otro lado, se realizó un análisis estadístico en el Software SPSS, donde se obtuvieron los resultados que permitieron medir el desempeño de las organizaciones en cuanto al cumplimiento de las acciones de Responsabilidad Social en las empresas de servicios de la Ciudad de Ocaña.

Las variables estudiadas se aprecian en la Tabla 1.

Tabla 1

Dimensiones de la RSE bajo la ISO 26000

\begin{tabular}{|c|c|}
\hline Variable & Descripción \\
\hline Gobernanza en la organización & $\begin{array}{l}\text { Evidencia los principios y fundamentos éticos en cada uno de los aspectos } \\
\text { concernientes a la operación de la empresa, su transparencia interna y } \\
\text { externa, así como su cumplimiento con el régimen legal }\end{array}$ \\
\hline Prácticas laborales & $\begin{array}{l}\text { Evidencia todas aquellas prácticas y políticas que involucran a los } \\
\text { trabajadores de la propia organización o a trabajadores subcontratados }\end{array}$ \\
\hline $\begin{array}{l}\text { Participación activa y desarrollo de la } \\
\text { comunidad }\end{array}$ & $\begin{array}{l}\text { Evalúa cómo la empresa contribuye al desarrollo y superación de las } \\
\text { comunidades inmediatas en donde opera y donde tenga intereses } \\
\text { comerciales }\end{array}$ \\
\hline Prácticas justas de operación & $\begin{array}{l}\text { Hacen referencia a la necesidad de que la organización tenga un } \\
\text { comportamiento ético en sus relaciones con otras organizaciones, así } \\
\text { como sus partes interesadas }\end{array}$ \\
\hline Medio ambiente & $\begin{array}{l}\text { Bajo esta dimensión se evalúa el respeto y cuidado del medio ambiente } \\
\text { en el desarrollo de la actividad empresarial }\end{array}$ \\
\hline Asunto de consumidores & $\begin{array}{l}\text { Evidencia la necesidad de que la empresa asuma responsabilidades con } \\
\text { sus consumidores, fomentando el consumo responsable y sostenible }\end{array}$ \\
\hline Derechos Humanos & $\begin{array}{l}\text { Mide el cumplimiento del desarrollo organizacional y su incidencia en el } \\
\text { respeto y la promoción de los derechos humanos, de sus empleados, } \\
\text { clientes, proveedores y personal de apoyo }\end{array}$ \\
\hline
\end{tabular}

Fuente: Las autoras a partir de Secretaría SIS/ABNT (2009)

\section{Resultados}

\subsection{Prácticas de responsabilidad social empresarial en las Mipymes del sector servicios}

La Responsabilidad Social bajo los lineamientos de la ISO 26000, identifica Siete Dimensiones fundamentales que toda organización debe considerar para llevar a cabo buenas prácticas para beneficio propio y de sus diferentes grupos de interés, donde la organización debe centrar su atención a la hora de actuar responsablemente (ISO $26000,2014)$. A partir del análisis de los resultados de la investigación, de acuerdo a las respuestas emitidas por los 117 Directivos de las diferentes empresas del sector Servicios objeto de estudio se encontraron los resultados que se aprecian en la Tabla 2. 
Tabla 2

Resultados de la RSE bajo la ISO 26000

en las empresas del sector servicios

\begin{tabular}{|c|c|c|}
\hline \multirow{2}{*}{ Dimensiones } & \multicolumn{2}{|c|}{ Descriptivos } \\
\hline & Media & Des. Est \\
\hline 1. Gobernanza & 3.56 & 1.10 \\
\hline 2. Prácticas laborales & 2.88 & 1.33 \\
\hline 3. Participación activa y desarrollo de la comunidad & 3.19 & 0.98 \\
\hline 4. Prácticas justas de operación & 4.35 & 0.63 \\
\hline 5. Medio ambiente & 3.15 & 1.04 \\
\hline 6. Asunto de consumidores & 3.80 & 0.82 \\
\hline 7. Derechos humanos & 3.50 & 1.02 \\
\hline
\end{tabular}

Fuente. Autores del proyecto

\section{Dimensión Gobernanza en la Organización}

Al observar los datos relacionados a la dimensión gobernanza a través de la medición del software estadístico SPSS se puede apreciar una media de 3.56, identificándose un alto compromiso en la formalidad legal, el pago de sus impuestos y la comunicación de la información contable a sus grupos de interés, evidenciándose que las organizaciones bajo estudio direccionan sus estrategias al cumplimiento de las prácticas legales del país, consiguiendo beneficios y oportunidades de sostenibilidad en los mercados. Es así como el estudio realizado por la Universidad Externado de Colombia en el año 2014, "La Informalidad Empresarial en ColombiaCaracterización” precisó que la formalización empresarial genera visibilidad en el mercado local y extranjero, trascendiendo positivamente en el incremento de las ventas.

Sin embargo, presentan aspectos críticos en lo referente a su planeación estratégica, direccionada en forma intuitiva y no dentro del contexto de una estrategia organizacional, coincidiendo con el estudio realizado por Peñaranda y Manzano (2010) que determinaron que las empresas de servicios no desarrollan la función de planeación de manera formal ni tienen definida una misión al negocio.

\section{Dimensión prácticas laborales}

En la dimensión de prácticas laborales se observa una media de 2.88, evidenciándose que los aspectos evaluados no respaldan las buenas prácticas de RSE siendo el más preocupante el relacionado con los programas para estimular la innovación a través de incentivos a la generación de ideas de los colaboradores, lo que genera desmotivación, así como el desmejoramiento del ambiente laboral. Al no invertir de manera óptima en capacitación o desarrollo profesional se originan niveles bajos de competitividad. En este sentido cabe resaltar la premisa de Osorio (2005), quien considera que la competitividad y el ambiente de trabajo tienen una relación directamente proporcional, donde las personas en ambientes laborales adecuados, generan en los colaboradores motivación y creatividad, incluso haciendo que los colaboradores alinean sus propios valores con los de la organización.

\section{Dimensión participación activa y desarrollo de la comunidad}

Las empresas forman parte de la Comunidad donde operan y tienen interés en su bienestar, de ahí que deben contribuir a promover niveles más elevados de bienestar dentro de su área de influencia. Los resultados de la investigación muestran una media de 3.19, evidenciandose que efectivamente las empresas de Servicios de la Ciudad de Ocaña, reconocen a la comunidad como parte importante en sus procesos de toma de decisiones. 
Igualmente, estas se comprometen a dar preferencias a los productos y servicios locales contribuyendo al fortalecimiento del tejido empresarial. En base a esta estrategia, sus acciones deben estar direccionadas al involucramiento en programas de participación ciudadana, la paz y los valores democráticos, así como propender por el registro, control y seguimiento de los reclamos de la comunidad sobre el impacto de las operaciones. Por consiguiente las empresas deben estar en un constante monitoreo del entorno para la toma de decisiones (Hernandez- Fernandez, 2016).

\section{Dimensión prácticas justas de operación}

Desde la perspectiva de la ISO 26000 las prácticas justas de operación representan el comportamiento ético de una empresa al relacionarse con otras organizaciones, forjando relaciones positivas. En las empresas objeto de estudio se observa el cumplimiento de estas buenas prácticas como componente esencial en el desarrollo de su actividad presentando una media de 4.35 , contando con procedimientos claros y transparentes en la adquisición de bienes y servicios, honestidad en mensajes publicitarios y proveedores comprometidos con las prácticas de responsabilidad social empresarial.

\section{Dimensión medio ambiente}

En cuanto al cumplimiento de la Responsabilidad Social en la dimensión del Medio Ambiente en las empresas objeto de estudio presentan una media de 3.15, donde se puede apreciar que estas están comprometidas con el desarrollo de campañas internas al cuidado del medio ambiente en aspectos como: Consumo de agua y energía, diseño y desarrollo de sus productos y políticas para el adecuado uso de los residuos. En contraste con la falta de un plan de emergencia ambiental generada por la empresa y la baja implementación en programas de educación ambiental encaminados a mejorar la conciencia de los empleados y demás grupos de interés.

En el estudio realizado por Peña y Serra (2013), enfocado a las empresas del sector turístico, evidencia en su análisis al entorno ambiental, que estas empresas están comprometidas con la mejora continua del desempeño ambiental, pero no se encuentran evidencias sobre Programas de Gestión Ambiental, Planes de Riesgos e Informes Ambientales. Lo que permite inferir que a pesar de que las empresas realizan esfuerzos para minimizar el impacto ambiental, estos no se encuentran establecidos en programas que direccione el accionar de la organización en este sentido. La responsabilidad social empresarial se visibiliza de forma importante cuando se enfoca hacia aspectos ambientales y proyectos de desarrollo sostenible (González, Manzano y Torres, 2018).

\section{Dimensión asunto de consumidores}

Desde la perspectiva de los consumidores, la ISO 26000 argumenta la responsabilidad social de las empresas que proveen productos y servicios a consumidores, y enfatiza de la responsabilidad que estas tienen hacia ellos. En base a esto, se observa que las empresas del sector Servicios de la Ciudad de Ocaña, presentan una media de 3.80 , ya que contemplan programas de educación hacia el respeto por el cliente y consumidor, incrementando la imagen positiva y la fidelidad que tienen sus usuarios. Igualmente el estudio demuestra que las empresas son socialmente responsables en cuanto a garantía y precios de sus productos y servicios. En contraste al cumplimiento de esta dimensión, se encuentra la falta de un procedimiento formal para conocer la satisfacción de sus clientes y consumidores que le apunta al mejoramiento de las buenas prácticas con los consumidores.

\section{Dimensión derechos humanos}

Ser responsable socialmente implica respetar los derechos humanos como prioridad en la gestión empresarial; en el mundo de hoy los derechos humanos son una parte intrínseca de las buenas prácticas de responsabilidad social, de ahí que el sector empresarial bajo estudio presenta una media de 3.5 encontrándose en consonancia con algunas de estas buenas prácticas, a pesar de que un gran número de empresas no cuentan con normas escritas que prohíban y sancionen prácticas discriminatorias por credo, género, edad, raza, discapacitados en los 
procesos de admisión y promoción interna, pero tratan de incluir dentro de su hacer empresarial a la diversidad poblacional, respeto a la privacidad de sus empleados, trato equitativo e igualdad de oportunidades.

\subsection{Análisis de varianza de las dimensiones de la RSE de las empresas del sector servicios}

Las empresas bajo estudio fueron divididas de acuerdo a su tamaño en tres grupos: 1-10 empleados, 11-50 empleados y de 51-200 empleados. En la Tabla 3 mediante el software estadístico SPSS, se analizan cada una de las dimensiones contempladas en la ISO 26000 evidenciándose una diferencia entre las medias de las diferentes dimensiones dependiendo del tamaño de la empresa. Por lo tanto se hizo necesario determinar a través de un análisis de varianza (ANOVA), si las diferencias encontradas son estadísticamente significativas o si esas diferencias entre las medias son atribuibles solo al azar.

Como todos los niveles de significancia están por debajo de Sig=0.05 (Ver Tabla 3), se pudo concluir que existe una diferencia estadísticamente significativa en la media de las diferentes dimensiones influenciadas por el tamaño de la empresa. Para lo cual se hizo necesario identificar que grupos son los que tienen medias diferentes entre sí, empleando la prueba de comparaciones múltiples Scheffé, donde se pudo constatar que las diferencias estadísticamente significativas están concentradas principalmente en las empresas de 1 a 10 empleados con las otras empresas de mayor tamaño.

Tabla 3

Estadísticos descriptivos y ANOVA de las dimensiones y tamaño de la empresa

\begin{tabular}{|c|c|c|c|c|}
\hline DIMENSIONES & & $\mathrm{N}$ & Media & Sig. \\
\hline \multirow{3}{*}{ Gobernanza } & $1,00^{*}$ & 93 & 3,2624 & \multirow[b]{3}{*}{,000 } \\
\hline & $2,00 * *$ & 16 & 4,6250 & \\
\hline & $3,00 * * *$ & 8 & 4,9000 & \\
\hline \multirow{3}{*}{ Practicas laborales } & 1,00 & 93 & 2,5634 & \multirow[b]{3}{*}{,000 } \\
\hline & 2,00 & 16 & 3,9250 & \\
\hline & 3,00 & 8 & 4,4250 & \\
\hline \multirow{3}{*}{ Participacion_desarrollo } & 1,00 & 93 & 2,9656 & \multirow[b]{3}{*}{,000 } \\
\hline & 2,00 & 16 & 4,0250 & \\
\hline & 3,00 & 8 & 4,0750 & \\
\hline \multirow{3}{*}{ Practicas justas } & 1,00 & 93 & 4,2753 & \multirow[b]{3}{*}{015} \\
\hline & 2,00 & 16 & 4,7625 & \\
\hline & 3,00 & 8 & 4,3250 & \\
\hline \multirow{3}{*}{ Medio ambiente } & 1,00 & 93 & 4,2753 & \multirow[b]{3}{*}{015} \\
\hline & 2,00 & 16 & 4,7625 & \\
\hline & 3,00 & 8 & 4,3250 & \\
\hline \multirow{3}{*}{ Consumidores } & 1,00 & 93 & 3,6043 & \multirow[b]{3}{*}{,000 } \\
\hline & 2,00 & 16 & 4,7375 & \\
\hline & 3,00 & 8 & 4,2250 & \\
\hline \multirow{3}{*}{ Derechos humanos } & 1,00 & 93 & 3,2774 & \multirow[b]{3}{*}{,001 } \\
\hline & 2,00 & 16 & 4,0875 & \\
\hline & 3,00 & 8 & 4,2250 & \\
\hline
\end{tabular}

$* 1-10, * * 11-50, * * * 51-200$ empleados

Fuente: Los autores con el empleo del software SPSS

Los resultados demuestran que el tamaño de la empresa incide en la manera como las organizaciones gestionan la Responsabilidad Social, identificándose como las pequeñas empresas organizan las prácticas de responsabilidad social en forma intuitiva y no dentro del contexto de una estrategia empresarial. Fundación 
Prohumana (2006), expone que las Pymes son caracterizadas por prácticas de Responsabilidad Social Empresarial que nacen a partir de motivaciones propias de los directivos o propietarios de la empresa. Mientras que las empresas que cuentan con un número mayor de trabajadores incorporan las prácticas de Responsabilidad Social como un programa dentro de su gestión empresarial.

Por otro lado, las empresas objeto de estudio fueron divididas de acuerdo al nivel de estudio del gerente en 4 grupos de la siguiente forma: 1 (Bachiller/técnico),2 (Tecnólogo), 3 (Profesional) ,4 (Posgrado). En la Tabla 4 se analizan las diferencias en las medias encontradas y un análisis de varianza (ANOVA), para identificar si las diferencias son estadísticamente significativas.

\section{Tabla 4}

Estadísticos descriptivos y ANOVA de las Dimensiones

y nivel de Formación del Gerente

\begin{tabular}{|c|c|c|c|c|}
\hline \multicolumn{2}{|c|}{ DIMENSIONES } & $\mathrm{N}$ & \multirow{2}{*}{$\frac{\text { Media }}{2,7810}$} & Sig. \\
\hline Gobernanza & 1,00 & 42 & & \multirow{4}{*}{,000 } \\
\hline & 2,00 & 18 & 2,8889 & \\
\hline & 3,00 & 28 & 4,0000 & \\
\hline & 4,00 & 29 & 4,6828 & \\
\hline \multirow{4}{*}{ Praclaborales } & 1,00 & 42 & 2,0905 & \multirow[t]{4}{*}{, 000} \\
\hline & 2,00 & 18 & 2,0556 & \\
\hline & 3,00 & 28 & 3,1643 & \\
\hline & 4,00 & 29 & 4,2483 & \\
\hline \multirow{4}{*}{ Participacion_desarrollo } & 1,00 & 42 & 2,7571 & \multirow{4}{*}{,000 } \\
\hline & 2,00 & 18 & 2,6333 & \\
\hline & 3,00 & 28 & 3,2071 & \\
\hline & 4,00 & 29 & 4,1310 & \\
\hline \multirow{4}{*}{ Practicas justas } & 1,00 & 42 & 4,3524 & \multirow[t]{4}{*}{,770 } \\
\hline & 2,00 & 18 & 4,4778 & \\
\hline & 3,00 & 28 & 4,2857 & \\
\hline & 4,00 & 29 & 4,3103 & \\
\hline \multirow{4}{*}{ Medio ambiente } & 1,00 & 42 & 4,3524 & \multirow{4}{*}{,780 } \\
\hline & 2,00 & 18 & 4,4778 & \\
\hline & 3,00 & 28 & 4,2857 & \\
\hline & 4,00 & 29 & 4,3103 & \\
\hline \multirow{4}{*}{ Consumidores } & 1,00 & 42 & 3,4095 & \multirow{4}{*}{,000 } \\
\hline & 2,00 & 18 & 3,4778 & \\
\hline & 3,00 & 28 & 4,0357 & \\
\hline & 4,00 & 29 & 4,3448 & \\
\hline \multirow{4}{*}{ Derechos humanos } & 1,00 & 42 & 2,7476 & \multirow[t]{4}{*}{,000 } \\
\hline & 2,00 & 18 & 3,8778 & \\
\hline & 3,00 & 28 & 3,7214 & \\
\hline & 4,00 & 29 & 3,9517 & \\
\hline
\end{tabular}

Fuente: Los autores a partir del software SPSS

En el análisis de varianza se pudo apreciar que en las dimensiones gobernanza, prácticas laborales, asunto de consumidores, derechos humanos, participación activa y desarrollo de la comunidad los valores de significancia están por debajo de Sig=0.05 pudiendo concluir que el factor nivel de formación del gerente tiene un impacto positivo, es decir, la media aumenta en la medida que el gerente es profesional o posee postgrado, mientras que en las dimensiones prácticas justas y medio ambiente no está influyendo el nivel de formación del gerente para la diferencia de medias. 
Al realizar la prueba de comparaciones múltiples Scheffé, los resultados demuestran que en la medida que el gerente tenga un nivel de formación alto tiene la empresa una mejor responsabilidad social en sus diferentes dimensiones, en donde las principales diferencias se centran entre bachiller con profesional y posgrado. Este resultado es coherente con la investigación realizada por Ramírez, Moctezuma y González (2017) quienes afirmas que la cualificación proporciona herramientas para enfrentarse al nuevo panorama laboral y social, permitiendo a los individuos ser agentes de cambio y transformación social que posibilite fomentar una cultura comprometida con prácticas de Responsabilidad Social Empresarial.

\section{Conclusiones}

Se puede concluir que las prácticas de responsabilidad social de las empresas del sector servicios de la ciudad de Ocaña, centran su mayor compromiso de Responsabilidad Social Empresarial en la dimensión de Prácticas justas de operación, seguidas de la materia Asuntos de Consumidores, Gobernanza y Derechos Humanos. Los promedios más bajos lo presentan las dimensiones Prácticas laborales, Participación Activa y Desarrollo de la Comunidad y Medio ambiente.

En la dimensión gobernanza en la organización las empresas de servicios, presentan fortalezas en lo concerniente al cumplimiento de disposiciones legales y formalización empresarial, encontrándose que el 90\% está legalmente constituida. En lo relacionado con la planeación estratégica, estas tienen dificultades a la hora de definir la ruta y avanzar hacia el crecimiento y fortalecimiento que conlleve a lograr el cumplimiento de los objetivos empresariales.

Frente a las práctica laborales, las empresas de servicios no formalizan sus vínculos contractuales y no cumplen con las obligaciones exigidas por el sistema de seguridad social, pues solo el $45 \%$ de las organizaciones afilian a sus empleados. La formación al personal no es una preocupación primordial del empresario, pues el $40 \%$ nunca capacita a los empleados y ven la cualificación como un gasto y no como el desarrollo de capacidades que aumenten la productividad empresarial. En relación a dimensión Participación Activa y Desarrollo de la Comunidad, estas direccionan su accionar en la maximización de la riqueza, apartándose de sus compromisos con la comunidad donde desarrolla sus operaciones.

Otro factor clave en la investigación lo constituyen las prácticas justas de operación, donde las Mipymes administran bajo la confidencialidad la información de los clientes y realizan mejoras en sus procesos buscando la sostenibilidad. Así mismo es importante destacar que en el contexto ambiental, las Mipymes realizan la operación empresarial bajo la premisa de las buenas prácticas ambientales para crecer sustentablemente, sin embargo estas deben enmarcar sus procesos para generar programas estructurados para la conservación y el cuidado del medio ambiente.

En cuanto a la dimensión asunto de consumidores, estas empresas consideran el cliente como el factor clave para el desarrollo y sostenimiento empresarial pero descuidan su fidelización, pues el $64 \%$ manifiestan no conocer la satisfacción del cliente frente al servicio. Por último en lo referente a los derechos humanos, estas empresas entienden y conciben la trascendencia del respeto y la promoción de los derechos humanos los cuales se encuentran inmersos en la relación con sus grupos de interés, no obstante no se encuentran estructurados en manuales o guías.

Finalmente, se puede concluir a través del análisis de varianza (ANOVA) que el tamaño de la empresa, el nivel de formación de los directivos y sus competencias gerenciales inciden de manera positiva en la gestión de la Responsabilidad Social, al observar que a mayor nivel de formación mayor es la comprensión de la importancia de la Responsabilidad Social al interior y exterior de sus empresas. 


\section{Referencias bibliográficas}

Carroll, A. B. (1979). A Three-dimensional Conceptual Model of Corporate Performance. Academy of Management Review, 4 (4), 497-505.

Comisión de las Comunidades Europeas (2001). Libro Verde: fomentar un marco europeo para la responsabilidad social de las empresas, documento COM: Bruselas.

Davis, K. (1960). Can Business afford to ignore social responsibilities. California Mangement Review, 2, 70-76.

Drucker, P. (1984). The new meaning of corporate social responsibility. California Management Review, 26 (2), 53-63

Drucker, P. (1993). The Post capitalist Society. New York: Harper Business.

Friedman, M. (1962). The Social Responsibility of Business is to Increase Its Profits. The New York Times Magazine.

Fundación Prohumana (2006). Responsabilidad Social en Chile: Una mirada desde los trabajadores.

González, Y., Manzano, O., Torres, M. (2018). Categorías de análisis para un Plan de Responsabilidad Social Universitaria. Bogotá: Sello Editorial UNAD. Recuperado de http://hemeroteca.unad.edu.co/index.php/book/issue/view/276

Hernandez Fernandez, L. (2016). Entorno y Empresa. Revista de Ciencias Sociales, 6-7.

Hernández, R., Fernández, C., \& Baptista, M. d. (2010). Metodología de la investigación (Quinta edición). México: McGRAW-HILL.

Hernández- Álvarez, E. A., \& Mendoza-Paredes, J. M. (2016). Responsabilidad Social Empresarial para Mipymes: un Escenario de Cumplimiento Metodológico para el Desarrollo Sostenible. Revista de Economía \& Administración, 71-82. Recuperado de https://ideas.repec.org/a/col/000156/015094.html

ISO 26000 (2014). Descubriendo la ISO 26000. Recuperado de: https://www.iso.org/files/live/sites/isoorg/files/store/sp/PUB100258_sp.pdf

Keith D. (1960). The Case for and against Business Assumption of Social Responsibilities. The Academy of Management Journal, $16(2$ ), 312-322.

Marquina-Feldman, P. \& Reficco, E. (2015). Impacto de la responsabilidad social empresarial en el comportamiento de compra y disposición a pagarde consumidores bogotanos. Revista Estudios Gerenciales, 373-382. Recuperado de: http://www.scielo.org.co/pdf/eg/v31n137/v31n137a03.pdf

McGuire, J. (1963). Business and society. New York: Estados Unidos: McGraw-Hill.

Osorio, M. (2005). Empresa y ética. La Responsabilidad Social Corporativa. Madrid.

Peñaranda, M. \& Manzano, O (2010). Una mirada al sector terciario como dinamizador del desarrollo regional. Revista Ingenio. 3 (2), 1-10.

Peña, D., \& Serra, A. (2013). La práctica de la responsabilidad social empresarial. Estudio de caso en el sector turístico. Revista de Ciencias Administrativas y Sociales, 23(49), 101-113. Obtenido de https://www.redalyc.org/pdf/818/81828691008.pdf 
Ramírez, Y., Moctezuma, M. y González, O. (2017). Importancia de la responsabilidad social en la formación de los futuros profesionales. Recuperado de https://www.uv.mx/iiesca/files/2017/10/07CA201701.pdf

Rojas, N., \& Madero, S. (2018). La Responsabilidad Social Corporativa: Contexto Histórico y Relación con las Teorías Administrativas. Revista Conciencia Tecnológica. Recuperado de: https://www.redalyc.org/jatsRepo/944/94455712001/html/index.html\#redalyc_94455712001_ref27

Secretaría SIS/ABNT (2009). Guia sobre Responsabilidad Social. Recuperado de: https://www.icesi.edu.co/blogs/paoladministradora/files/2012/06/ISO26000.pdf

Strandberg, L. (2010). La medición y la comunicación de la RSE: indicadores y normas. Cuadernos de la Cátedra "La Caixa" de Responsabilidad Social de la Empresa y Gobierno Corporativo, (9). Recuperado de: https://media.iese.edu/research/pdfs/ST-0322.pdf

Valotto, G. (2007). Evolución del sector servicios y el comercio internacional de servicios [en línea]. Disponible en: http://www.eumed.net/libros/2010a/673/El\%20Avance\%20del\%20Comercio\%20Internac ional\%20de\%20Servicios.htm, consultado el 16 de junio de 2011.

Vives, A. (2004). The Role of Multilateral Development Institutions in Fostering Corporate Social Responsibility, Sustainable Development Department, Technical Papers Series, Washington, D.C.: Inter-American Development Bank.

Wood, D. (1991). Corporate social performance revisited. Academy of Management Review, 16 (4), $691-718$.

Esta obra está bajo una Licencia Creative Commons Attribución-NoCommercial 4.0 International

\section{(cc) BY-NC}

